\title{
Original
}

\section{Synergistic Effect of Tolerogenic Dendritic Cells and Etanercept on a Collagen-induced Arthritis Animal Model}

\author{
Sang Youn Jung ${ }^{1) *}$, Kil-Sang Cho ${ }^{2) *}$, Doo-Rye Jang ${ }^{2) *}$, Jun-Ho Lee ${ }^{2,3)}{ }^{*}$, So-Yeon Choi ${ }^{2)}$, Sei-Hee Byun ${ }^{4)}$, Soo-Yeoun Park ${ }^{4)}$, Nam-Chul \\ Jung $^{3)}$, Jinjung Choi ${ }^{1)}$, Kwang-Hoe Chung ${ }^{2)}$, Jin-ah Jang ${ }^{5)}$, Jun-Eui Park ${ }^{5)}$, Hyun Soo Lee ${ }^{3)}$ and Dae-Seog Lim ${ }^{2)}$ \\ ${ }^{1)}$ Division of Rheumatology, Department of Internal Medicine, Bundang CHA Medical Center, Gyeounggi-do, Republic of Korea \\ 2) Department of Biotechnology, CHA University, Gyeonggi-do, Republic of Korea \\ 3) Pharos Vaccine Inc., Gyeonggi-do, Republic of Korea \\ 4) Aprogen Pharmaceuticals Inc., Gyeonggi-do, Republic of Korea \\ 5) JW CreaGene Research Institute, JW CreaGene Inc., Gyeonggi-do, Republic of Korea
}

(Accepted for publication, June 15, 2019)

\begin{abstract}
The aim of this study was to determine the potential for an additive effect from combining treatments with tolerogenic dendritic cells (tDCs) and etanercept (ETN) in a case of established type II collagen-induced arthritis (CIA). CIA mice were treated with type II collagen-pulsed tDCs either alone or in combination with ETN. We compared the effects of monotherapy with tDCs or ETN with the effect of combination treatment on the resultant rheumatoid arthritis using histopathological analyses and measurements of paw volumes, regulatory $\mathrm{T}$ cell populations, and cytokine levels. The clinical and histologic analysis revealed an additive effect from the combination therapy. Monotherapy with tDCs showed a tendency to ameliorate arthritis symptoms, while monotherapy with ETN reduced the paw swelling at 3 days after administration, but was not effective toward the end of the experimental period. The combined treatment group also showed a marked inducement of the regulatory $\mathrm{T}$ cells and Th2 cytokines that are affected by tDCs compared with those of either untreated or ETN-treated CIA mice. ETN administered in combination with tDCs provided a substantially higher level of clinical benefit at the cellular level to CIA mice than the administration of either of these agents alone.
\end{abstract}

Key words: Combination therapy, Etanercept, Regulatory T cells, Tolerogenic dendritic cells

\section{Introduction}

Rheumatoid arthritis (RA) is a systemic joint disease that occurs as a chronic inflammatory disorder that is characterized by cartilage destruction $^{1)}$. RA is characterized by the infiltration of leukocytes into the synovium and hyperplasia of the resident synoviocytes. The pathogenesis of RA assumes that the disease is driven by arthritogenic antigen-specific $\mathrm{T}$ cells that perpetuate the chronic inflammatory response $\mathrm{e}^{2,3)}$.

Biological products such as etanercept (ETN), which modulate the level of this pro-inflammatory cytokine, have been marketed and their efficacies have been demonstrated in patients who fail to respond adequately to conventional therapies ${ }^{4,5)}$. A growing body of evidence in clinical trials, however, is showing that disease remission can be achieved and maintained better when biologic agents are used earlier in the disease rather than later ${ }^{6-8)}$. The same findings have been confirmed in clinical practice where early intervention, $<3$ months, was the strongest predictor for a successful remission".

Tolerogenic dendritic cells (tDCs) play an important role in inducing peripheral tolerance via specific mechanisms, including activation of regulatory $\mathrm{T}$ (Treg) cells, suppression of effector $\mathrm{T}$ cells, and negative

\footnotetext{
* Sang Youn Jung, Kil-Sang Cho, Doo-Rye Jang, and Jun-Ho Lee contributed equally in this study.

Correspondence to: Dr. Dae-Seog Lim, Department of Biotechnology, CHA University, 335 Pangyo-ro, Bundang-gu, Seongnam, Gyeonggi-do 13488, Republic of Korea; Tel: +82-10-2770-4777; Fax: +82-31-881-7228; E-mail address: dslim@cha.ac.kr
}

modulation of Th1/Th2 immune responses ${ }^{10-12)}$. Treg cells suppress the function of conventional $\mathrm{T}$ cells, limiting the generation of inappropriate or excessive immune activation including the release of suppressor cytokines ${ }^{13,14)}$. Treg cells suppress the function of conventional $\mathrm{T}$ cells, limiting the generation of inappropriate or excessive immune activation including the release of suppressor cytokines. Treg cells also display important functions in the pathological process of $\mathrm{RA}^{15-17)}$. The use of tDCs for immunotherapy is an attractive approach to treating autoimmune diseases in an Ag-specific manner, which are associated with systemic immunosuppression and adverse side effects ${ }^{18,19)}$.

The purpose of the present study was to clinically investigate a combined treatment with the specific-cytokine inhibitor ETN and tDCs to determine its effect on a mouse model of type II collagen-induced arthritis (CIA).

\section{Mice}

\section{Materials and methods}

The protocols for the use of animals in these studies were approved by the Institutional Animal Care and Use Committee (IACUC) of CHA University (Project No. IACUC140043). All experiments were carried out in accordance with the approved protocols. The study used female DBA1/J mice (6-8 weeks of age, each weighing 15-18 g) purchased from Central Lab. The mice were cared for by Animal, Inc. (Seoul, Republic of Korea). 


\section{Drugs and reagents}

Etanercept, Recombinant (Enbrel; Pfizer limited, Ramsgate Road, UK), was used in this study. For the in vivo study, Enbrel was dissolved in saline for subcutaneous (s.c.) injection. Samples of type II collagen from chicken sternal cartilage, Freund's adjuvant Complete and Freund's adjuvant Incomplete, were purchased from Sigma Aldrich (St. Louis, MO, USA). RPMI1640 with HEPES (Lonza, Walkersville, MD, USA), FBS, certified, U.S. origin (Gibco, Life Technologies, Grand Island, NY, USA), antibiotics-antimycotics (Gibco), 2-mercaptoethanol (Gibco), recombinant mouse (rm) GM-CSF and IL-4 (JW CreaGene, Gyeonggi, Korea), lipopolysaccharide (Sigma) and rm TNF- $\alpha$ (BD Bioscience, Mountain View, CA, USA) were used in the cell culture. The ELISA kits for mouse IL-12p70, IFN- $\gamma$, IL-4, IL-10, and TNF- $\alpha$ were the products of $\mathrm{BD}$ and Biolegend (CA, USA). Anti-mouse PE-conjugated CD11c (HL3), CD40 (3/23), CD80 (16-10A1), I-Ad/I-Ed (2G9), CD274 (HK5.3), FITC-conjugated CD4 (GK1.5), CD14 (rmC5-3), CD54 (3E2), H-2Db (28-14-8), CD86 (GL1), and APC-conjugated CD25 (PC61.5) monoclonal antibodies were purchased from BD Bioscience. For intracellular staining, cells were fixed/permeabilized using an intracellular staining kit (eBioscience, CA, USA).

\section{Generation of bone marrow-derived DCs}

DCs were generated from bone marrow progenitors obtained from DBA/1 J mice, as previously described, with some modifications ${ }^{20)}$. Briefly, bone marrow cells were cultured in RPMI1640 with HEPES, $10 \%$ FBS, antibiotics-antimycotics, $55 \mathrm{nM} 2-\mathrm{ME}, 20 \mathrm{ng} / \mathrm{ml} \mathrm{rmGM}-\mathrm{CSF}$ and $2 \mathrm{ng} / \mathrm{ml} \mathrm{rmIL}-4$. Cells were maintained at $37^{\circ} \mathrm{C}$ under an atmosphere containing $5 \% \mathrm{CO}_{2}$. On days 3 and 6 , half of the culture medium was replaced with fresh medium containing the same concentrations of GM-CSF and IL-4. On day 7, immature DCs (imDCs) were allowed to mature DCs (mDCs) by exposure to $1 \mu \mathrm{g} / \mathrm{ml}$ LPS for $24 \mathrm{~h}$. On day 8 , tolerogenic DCs (tDCs) were generated by treating the imDCs with 10 $\mathrm{ng} / \mathrm{ml} \mathrm{rmTNF}-\alpha$ for $4 \mathrm{~h}$. At that point, all DCs (imDCs, tDCs, and mDCs) were simultaneously harvested and examined in further studies.

\section{Induction of CIA and treatment}

Type II collagen was dissolved overnight in $0.05 \mathrm{M}$ acetic acid at $4^{\circ} \mathrm{C}$ and then emulsified in an equal volume of complete Freund's adjuvant. To induce CIA, DBA/1J mice were immunized (subcutaneously into the base of the tail) with $100 \mu \mathrm{L}$ of emulsion containing $200 \mu \mathrm{g}$ of type II collagen. The mice were boosted with $200 \mu \mathrm{g}$ type II collagen emulsified in incomplete Freund's adjuvant on Day 21 post-primary immunization. To establish an enbrel-treated group, enbrel (3 $\mathrm{mg} / \mathrm{kg})$ was subcutaneously injected into CIA mice once every 3 days from days 21 to 36 . For the tDC-treated group, CIA mice were injected subcutaneously with $2 \times 10^{5}$ cells of type II collagen-pulsed tDCs on days 21 and 29 . The combination study group was subcutaneously injected with enbrel $(3 \mathrm{mg} / \mathrm{kg})$ once every 3 days from days 21 to 36 and with tDCs $\left(2 \times 10^{5}\right)$ on days 21 and 29.

\section{Assessment of arthritis severity}

An evaluation of the CIA severity was performed by two independent observers. The mice were evaluated every 2-3 days. The severity of arthritis was expressed on a mean arthritis index graded on a scale of $0-4$ : grade 0 , paws with no swelling and focal redness; grade 1, paws with swelling at the finger joint; grade 2, paws with mild swelling at the ankle or wrist joints; grade 3, paws with severe inflammation of the entire paws; grade 4, paws with deformity or ankylosis. The maximum total arthritis score that each mouse could receive in this system was 16 .
Five of the mice in each group were randomly chosen to be euthanized on Day 35. The spleen, inguinal lymph nodes, and paws were isolated and immune status examined.

\section{Histopathological analysis}

Paws from each group were fixed with 10\% neutral-buffered formalin solution and decalcified with $15 \%$ EDTA solution at day 52 after first immunization. Paraffin-embedded samples were sectioned and stained with hematoxylin and eosin (HE). The severity of the arthritis in the joints was graded from $0-5$ according to the intensity of the mononuclear cell infiltration, cartilage destruction, bone erosion, and pannus formation. The grades were as follows: grades 0 , normal ankle joint; grade 1, normal synovium with occasional mononuclear cells; grade 2, definite arthritis with slight infiltrations of cells and cartilage flanking; grade 3, clear hyperplasia of the synovium and dense infiltration with mononuclear cells and cartilage cleft; grade 4, highly dense infiltration with mononuclear cells and cartilage erosion; grade 5, severe synovitis with pannus and erosion of the articular cartilage and subchondral bones. The sum of the scores was used to express the severity of the arthritis for one joint (maximum severity score 15).

\section{Cytokine measurement}

The levels of interleukin-1 $\beta$ (IL-1 $)$, IL-10, IL-12p70, interferon(IF$\mathrm{N})-\gamma$, IL-17A, TNF- $\alpha$, and transforming growth factor (TGF)- $\beta 1$ (BD Bioscience) were measured using the supernatant of cultures containing either lymphocytes $\left(2 \times 10^{6}\right.$ cells $\left./ 2 \mathrm{ml}\right)$ or DCs $\left(1 \times 10^{6}\right.$ cells $\left./ 2 \mathrm{ml}\right)$ alone using commercially available ELISA kits, according to the manufacturer's instructions.

\section{Flow cytometry analysis}

Fluorescently conjugated monoclonal antibodies (mAbs) were used to examine the phenotype of the DCs and lymphocytes. Briefly, cells (1 $\left.\times 10^{5}\right)$ were incubated in FACS buffer $(0.2 \%$ BSA, $0.02 \%$ sodium azide in PBS) at $4{ }^{\circ} \mathrm{C}$ for 20 min along with the following $\mathrm{mAbs}$ : PE-conjugated CD11c (HL3), CD40 (3/20), CD80 (16-10A1), I-A /I-E ${ }^{\mathrm{d}}$ (2G9), FITC-conjugated CD4 (GK1.5), CD14 (rmC5-3), CD54(3E2), H-2D ${ }^{\mathrm{b}}$ (28-14-8), CD86 (GL1), and APC-conjugated CD25 (PC61.5). For intracellular staining, cells were fixed/permeabilized using an intracellular staining kit (BD Bioscience) or the Foxp3 staining buffer set (eBioscience) and then stained PE-conjugated IFN- $\gamma$ (XMG1.2), IL-17A (TC1118H10), and Foxp3 (FJK-16s) antibodies. After staining, cells were washed with FACS staining buffer and examined in a FACSCalibur flow cytometer (Becton Dickinson, Mountain View, CA, USA). Data were analyzed using FlowJo software (TreeStar, Inc., San Carlos, CA, USA).

\section{Co-culture of T lymphocytes and DCs}

Splenocytes were isolated from DBA/1J mice and disaggregated in RPMI 1640 medium. $\mathrm{CD}^{+} \mathrm{T}$ cells were isolated by passing the splenocytes through a nylon wool column. Cells were co-cultured at a DC:lymphocyte ratio of $1: 10$. Purified $\mathrm{CD}^{+} \mathrm{T}$ cells $\left(1 \times 10^{6}\right.$ cells $\left./ \mathrm{ml}\right)$ were used as responders and DCs $\left(1 \times 10^{5}\right.$ cells $\left./ \mathrm{ml}\right)$ were used as stimulators. Cells were co-cultured at $37^{\circ} \mathrm{C}$ for $72 \mathrm{~h}$ in $2 \mathrm{ml}$ of RPMI 1640 supplemented with $10 \%$ FBS.

\section{Statistical analysis}

Statistical analysis was performed using GraphPad software (GraphPad Prism v5.0; GraphPad Software Inc., San Diego, California, USA). Data were examined using one-way ANOVA followed by a Newman-Keuls test. Results were expressed as the mean $\pm \mathrm{SEM}$. A $p$-value 
(a)

imDC

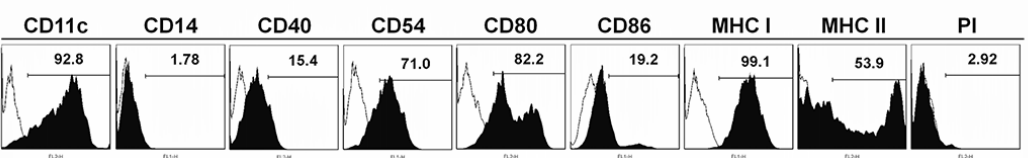

tDC

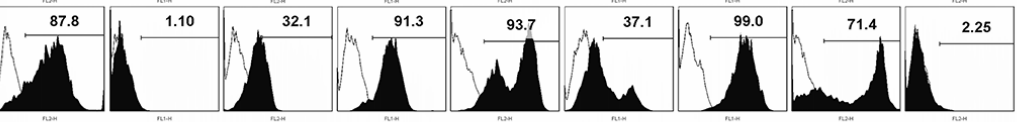

$\mathrm{mDC}$
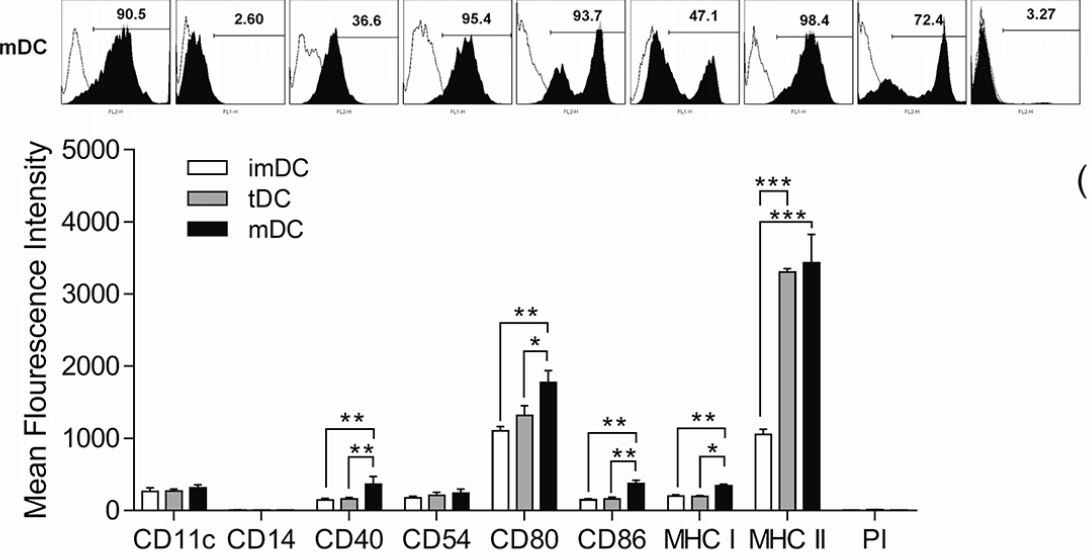

(b)

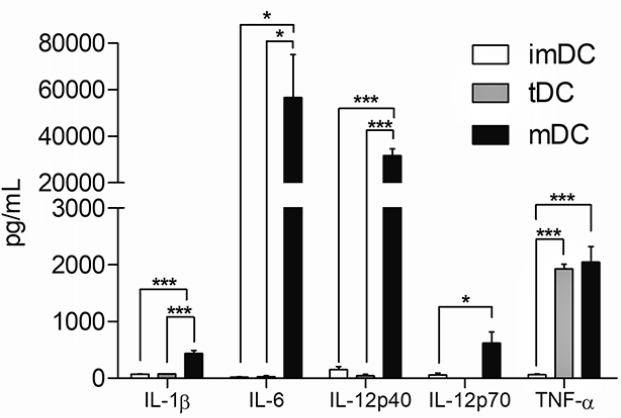

(c)
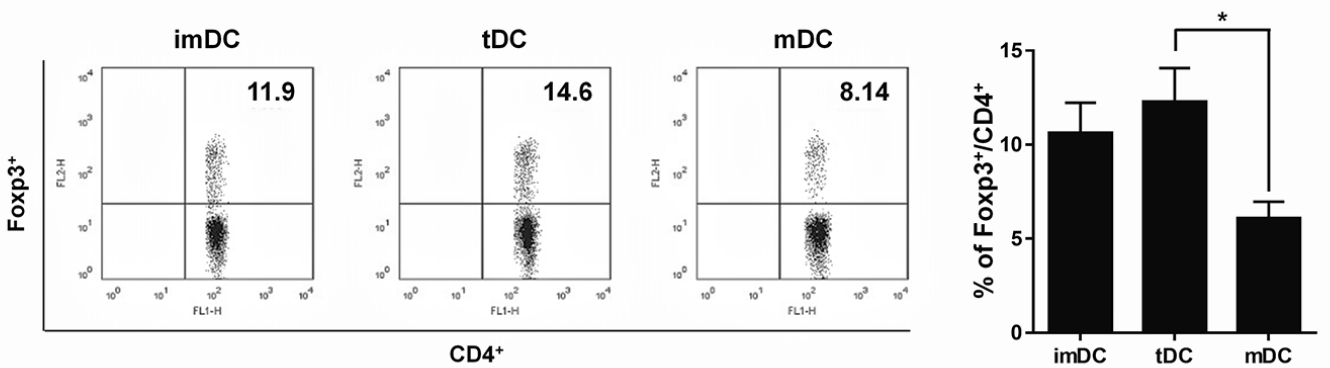

(d)

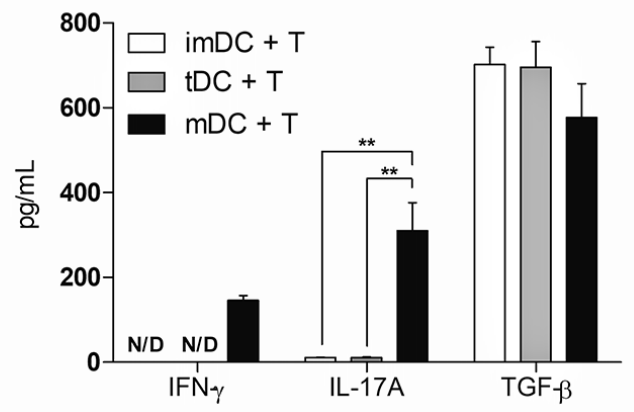

Figure 1. Characterization of DCs. (a) DC subsets (imDCs, tDCs, and $\mathrm{mDCs}$ ) were stained with the fluorescently-conjugated antibodies and analyzed by flow cytometry. Data are presented as histograms. (b) Cytokines in the culture supernatants of DCs were analyzed by ELISA ( $\mathrm{n}=5$ independent DC preparations). (c) Each DC subset was co-cultured with the splenocytes for $72 \mathrm{hr}$. The stimulator:responder ratio was 1:10. To identify Treg cells, total cells were stained with anti-CD4, -CD25, and Foxp3 antibodies in Foxp3 staining buffer and then analyzed by flow cytometry. The bar graph shows the percentage of Foxp $3^{+} \mathrm{CD} 4{ }^{+} \mathrm{CD} 25^{+}$cells. (d) Cytokine levels in the supernatants after $72 \mathrm{hr}$ of co-culture were measured by ELISA. All data are expressed as the mean \pm SEM $(n=10$ independent experiment performed in triplicate). $* P<0.05, * * P<0.01$; $* * * \mathrm{P}<0.001$.

of $<0.05$ was considered significant.

\section{Results}

Therapeutic effect of the combined treatment on the arthritis severity of CIA mice

The tolerogenic DCs (tDCs) were well characterized by a lower ex- pression of the co-stimulatory molecules, CD80, CD86, and CD40, compared with mature DCs (mDCs) (Fig. 1a). Cytokine production is an important mechanism by which DCs regulate immune responses, and tDCs produce lower levels of the inflammatory cytokines (IL-1 $\beta$, IL-6, IL-12p40, and IL-12p70) compared with mDCs (Fig. 1b). We next performed a series of functional co-culture experiments to investigate the 
(a)

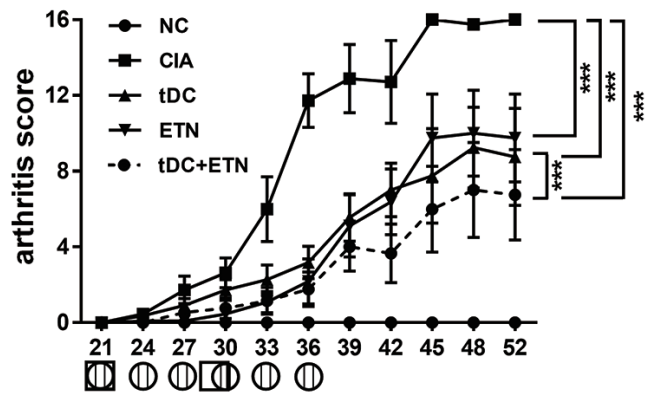

Days after first immunization

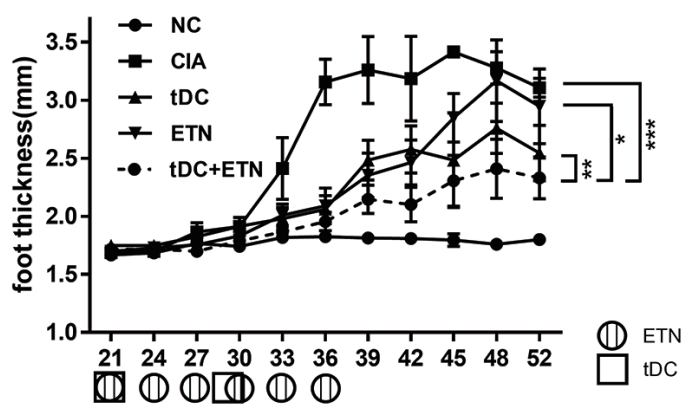

Days after first immunization

(b)
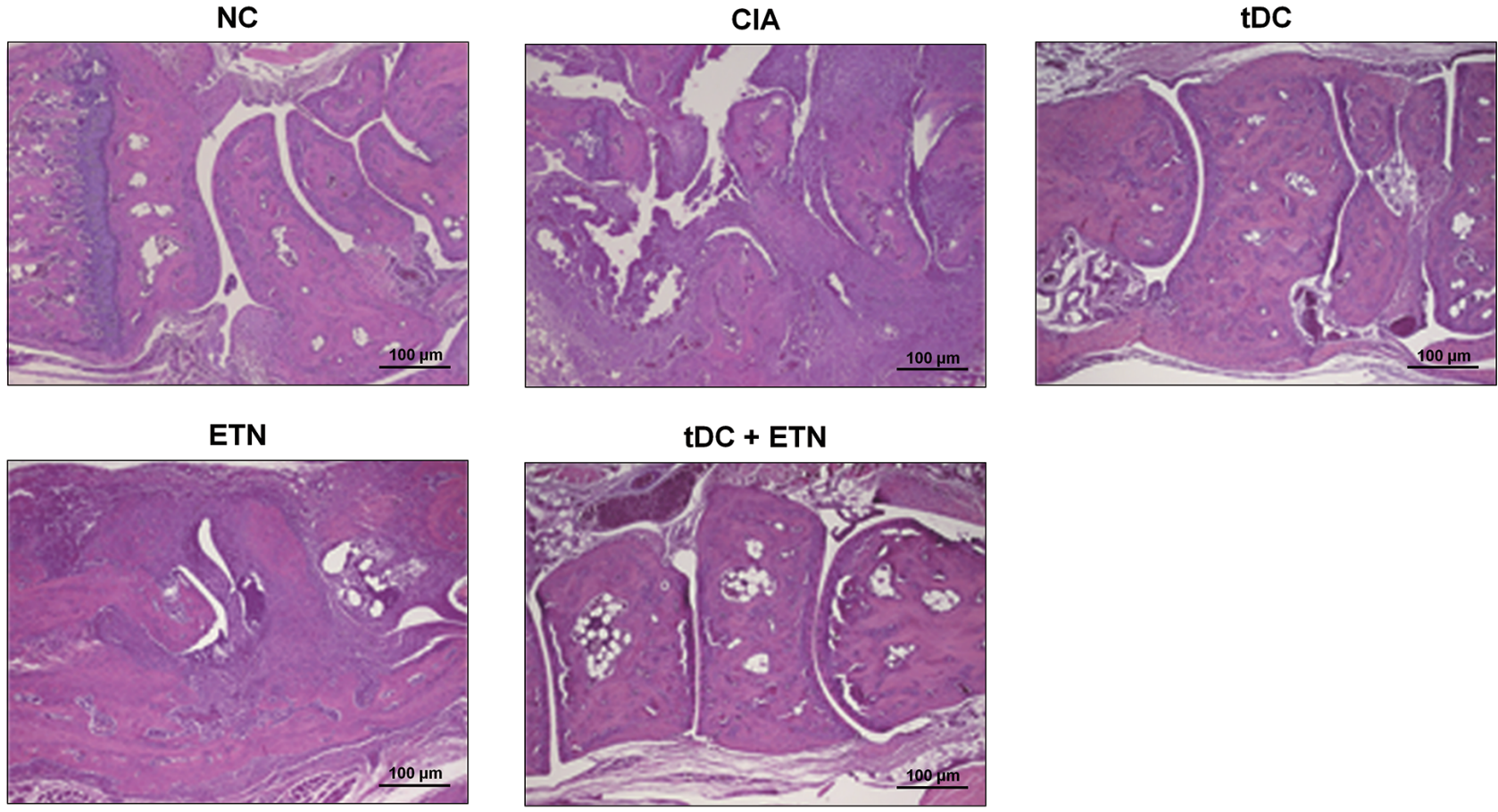

(c)

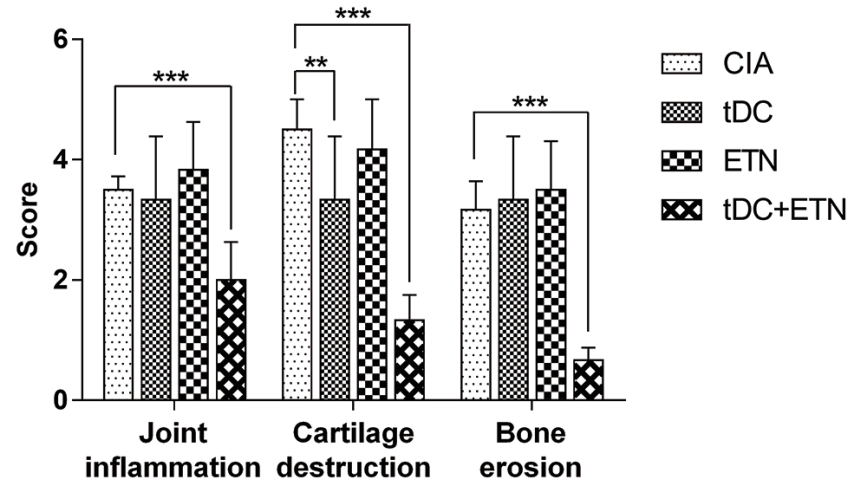

Figure 2. Effect of the combined treatment on arthritis severity in CIA mice. (a) The severity of disease was graded using a scale of 0-4 for each of the four paws by blind observers. See the Material and methods for details on the scale (left). The footpad thickness of each mouse was measured two or three times a week (right). Data show the means \pm SEM, $n=10$ mice per group. (b) Paws were removed from each group of mice at day 52 after first immunization, selected randomly, and fixed for 2 days in $4 \%$ formalin. Serial sections $(5 \mu \mathrm{m})$ were conducted and stained with hematoxylin and eosin (H\&E). Scale bars: $100 \mu \mathrm{m}$. Data shown are representative of at least 3 independent experiments. (c) Histopathological analysis of CIA. The histopathological score for an animal was calculated as the average of the scores of the left and right paw joints. Data show the means \pm SEM, $\mathrm{n}=5$ mice per group. $* \mathrm{P}<0.05,{ }^{*} * \mathrm{P}<0.01 ; * * \mathrm{P}<0.001$.

effect of tDCs on Treg cell expansion. The tDCs markedly increased the $\mathrm{CD} 4^{+} \mathrm{CD} 25^{+} \mathrm{Foxp}^{+}$Treg cell population (Fig. 1c). Additionally, com- pared with mDCs, tDCs inhibited the production of IFN- $\gamma$ and IL-17A (Fig. 1d). These results imply that the tDCs have the potential to induce 
(a)

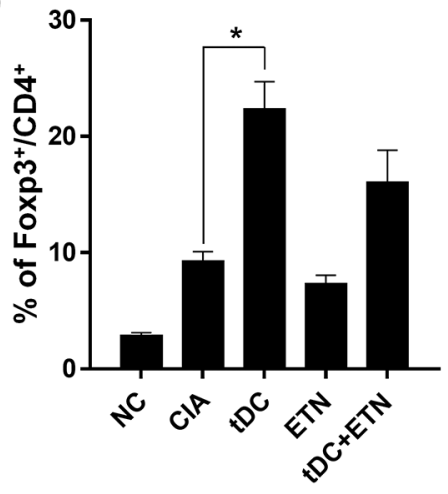

(b)

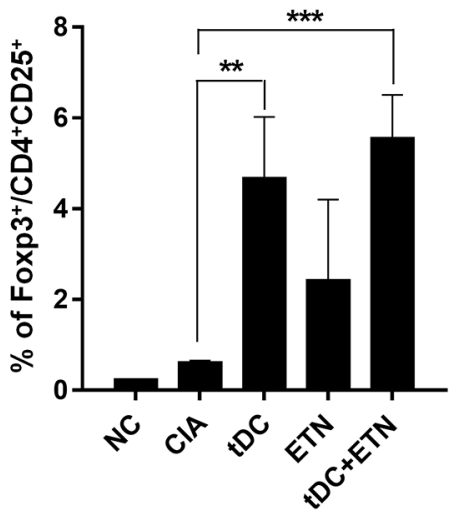

(c)
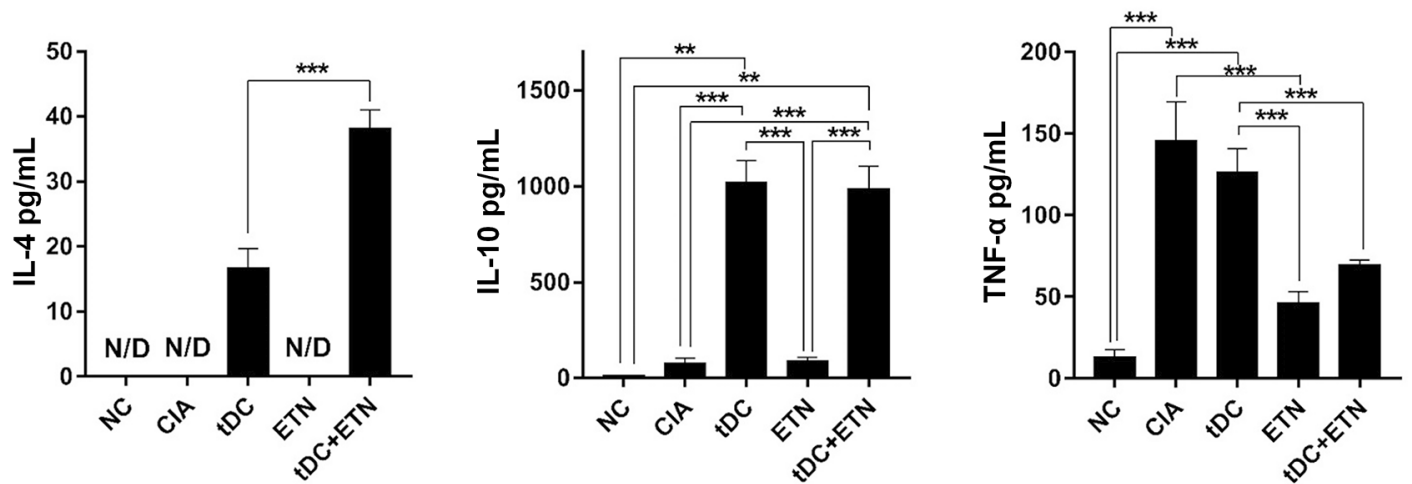

Figure 3. Ex-vivo immune status of combination treatment with tDC and ETN on CIA mice. Cells were isolated from the spleens and inguinal lymph nodes of each group of CIA mice and cultured with $25 \mu \mathrm{g} / \mathrm{mL}$ type II collagen for $72 \mathrm{hr}$. (a) On days $50, \mathrm{Ag}$-specific CD4 $4^{+} \mathrm{CD} 25^{+}$Foxp3 Treg cell population within the splenocytes population from CIA mice. (b) On days 50, Ag-specific $\mathrm{CD} 4^{+} \mathrm{CD} 25^{+}$Foxp $3^{+}$Treg cell population within the inguinal lymph node population from CIA mice. Data are representative five mice per group. (c) The levels of IL-4, IL-10, and TNF- $\alpha$ cytokines in culture supernatants were measured by ELISA. The bar data are expressed as the means \pm SEM, $\mathrm{n}=8$ mice per group. $* \mathrm{P}<0.05$, $* * \mathrm{P}<0.01 ; * * * \mathrm{P}<0.001$

tolerance when co-cultured with $\mathrm{T}$ cells.

To determine the therapeutic effect of the combination therapy in CIA, mice were separately treated with tDC and ETN, or with a combination of both. Then the changes in arthritis scores and paw thickness were recorded until day 50 from the first type II collagen immunization. The treatment with tDC alone resulted in a $45.31 \pm 5.12 \%$ inhibition of the paw swelling, and treatment with ETN alone resulted in a $39.06 \pm$ $4.64 \%$ inhibition of the paw swelling compared with CIA (PBS-treated group) (Fig. 2a-left). We investigated the effect of single treatments with tDC or ETN on day 35 with the effect of a combination of those treatments, and found that the single treatments had not maintained efficacy toward the end of the experimental period. In contrast, the combined treatment had significantly reduced paw swelling, and at the end of the experimental period, had resulted in a $57.81 \pm 4.78 \%$ inhibition of paw swelling (Fig. 2a-right).

\section{Therapeutic effect of the combined treatment on the injuries to joints in the CIA mice}

Mice were euthanized at day 50 after the first immunization, and the paws were examined. In the CIA mice, synoviocytes proliferated to multilayers, and articular cartilage showed destruction and was infiltrated with inflammatory cells. In mice given tDC or the combined treatment, the synovial hyperplasia and the pannus were significantly inhibited and the destruction of the articular cartilage was alleviated (Fig. $2 b)$. The inflammatory scores of the joints in the mice with CIA were in- creased compared with those of the normal mice. The combined treatment group experienced a significantly better inhibition of synovial inflammation than either the tDC or ETN groups. The values for cartilage destruction and bone erosion of the joints followed a trend similar to that of the inflammation scores (Fig. 2c).

Effects of the combined treatment compared with tDC or ETN alone on the induction of antigen-specific Treg cells and cytokine production in CIA mice

To confirm the in vivo effects of treatment with type II collagen-pulsed tDCs on the Treg cell population, splenocytes and lymph node cells from CIA mice were harvested at days 35 after the first immunization. Treg cells were markedly increased in the spleens of mice vaccinated with type II collagen-pulsed tDCs alone or in combination with ETN compared with those observed in other groups, whereas the increase in the Treg cells population in mice treated with ETN and non-treatment groups had not reached statistical significance level (Fig. $3 \mathrm{a}, \mathrm{b})$. On days 35 , splenocytes from the each mice group were cultured in stimulation with type II collagen and measured for Ag-experienced T cells production in the cytokine. The production of anti-inflammatory cytokines, IL-10 and IL-4, increased by significant levels, respectively, in the tDC treatment groups. In contrast, the CIA (PBS-treated group) and ETN-alone groups scarcely expressed any cytokines (Fig. 3c). At the same time, decreased production of TNF- $\alpha$ was observed in the supernatant of splenocytes from type II collagen-pulsed tDCs in combina- 
tion with ETN and ETN alone compared with those observed in other groups (Data not shown).

\section{Discussion}

TNF- $\alpha$ inhibitors are remarkably effective in the treatment of rheumatoid arthritis ${ }^{21)}$. These drugs have changed the treatment goals for RA to include not only the inhibition of joint destruction but also the induction of remission. Etanercept (ETN), like other TNF- $\alpha$ inhibitors such as adalimumab and infliximab, not only binds with high affinity to soluble and cell-associated TNF- $\alpha$ but also binds to lymphotoxin- $\alpha$ (known as TNF- $\beta)^{22)}$. It is possible that loss of efficacy in patients who have been switched from ETN to tocilizumab occurs because TNF- $\beta$ may be activated when treatment with ETN is stopped, and disease activity may worsen. In this combination study, we investigated ETN treatment alone on the day of arthritis onset. Treatment with ETN alone reduced paw swelling immediately after administration, but it had no effect on swelling at the end of the experimental period.

In recent reports and recommendations, therapy for RA has been required to change and be maintained to at least achieve low disease activity by composite scores, and, ideally, to attain remission ${ }^{9}$. Research in immune modulation has increased in recent years, and a particular subset of dendritic cells has been used to modulate immune responses. DCbased immunotherapy has been a primary consideration in the field of cancer therapy ${ }^{23)}$. However, some reports have demonstrated that DCs are able to induce not only immunity but also immune tolerance, depending on the stage of their maturation. In fact, some papers have demonstrated that tDCs have the potential for the treatment of autoimmune disease ${ }^{18,20,24)}$. Also, in vitro-generated tDCs, which were matured by TNF- $\alpha$ and subcutaneously injected into mice, are known to function in a tolerogenic fashion via the prevention of Th1- and Th17-dependent $\mathrm{RA}$, by inducing antigen-specific $\mathrm{CD} 4^{+} \mathrm{CD} 25^{+} \mathrm{Foxp}^{+} \mathrm{Treg}^{20,25)}$. A failure of Treg function has been implicated in the development of many autoimmune processes, whereas cellular therapy by adoptive transfer of Tregs has shown efficacy in these disorders. In addition, DCs regulate Treg homeostasis and vice versa. IL-10 is unique among cytokines because it can induce Foxp3 expression and Treg differentiation in the absence of DC. In the present study, we focused on the in vivo environment by which tDCs induce and control tolerance, particularly the function and differentiation of Tregs, which are crucial to containing autoimmunity and chronic inflammation.

Several studies have examined the association between the status of $\mathrm{T}$ cells, cytokines and the pathologic features of RA. Few reports have discussed whether tDC treatment exerts its therapy on the subsets of $\mathrm{T}$ cells in both central and peripheral lymphoid organs. Furthermore, the inhibition of TNF in patients with RA has promoted the emergence of a Treg cell population that could suppress Teff cells through IL-10-dependent pathways ${ }^{26)}$. In this regard, our study achieved an effective approach by inducing Treg cells with well-defined specificities, wherein these cells were retargeted to suppress arthritis caused by specific antigen pulsed-dendritic cells ${ }^{18,19)}$.

There are a number of conceivable reasons that can account for the efficacy of the combination therapy. One possibility suggested by our results was that the $\mathrm{tDC}$-alone and combined-treatment groups produced more IL-4 and IL-10 compared with the ETN-alone group. These results imply that tDCs have the potential to induce tolerance in CIA when vaccinated with DCs that have Ag specificity. We noted another possible explanation for the efficacy of the combination therapy when type II collagen pulsed-tDC therapy also markedly increased the levels of Foxp $3^{+}$Treg cells in the spleen and lymph nodes of mice in the tDC- alone and combination groups, implying that negative immune modulation-promoting Treg cells may contribute to the suppression of RA. In contrast, the CIA (PBS-treated group) and ETN-alone groups showed markedly low levels of Foxp $3^{+}$Treg cells in the spleen and lymph nodes on day 35 .

In conclusion, the tDC and ETN combination therapy reduced the development of arthritis and showed a continuous effect in the CIA model. Only this therapy exhibited a tendency to reduce synovial proliferation, bone destruction, and immune cell infiltration in CIA. These findings suggest that the induction of Treg cells is an important clinical benefit of immunosuppressive therapy.

In the present study, a combination therapy with tDC and ETN showed therapeutic action on CIA that was superior to either tDC or ETN monotherapies, which might have been related to the modification of inflammation mediators and the expression of regulatory $\mathrm{T}$ lymphocytes in CIA.

\section{Acknowledgements}

This research was supported by the Bio \& Medical Technology Development Program of the National Research Foundation, funded by the Korean government (grant number: NRF-2017M3A9C8062622) and a grant of the Korea Health Technology R\&D Project through the Korea Health Industry Development Institute (KHIDI), funded by the Ministry of Health \& Welfare, Republic of Korea (grant number: HI13C0918).

\section{Conflict of Interest}

The authors have declared that no COI exists.

\section{References}

1. Goronzy JJ and Weyand CM. Rheumatoid arthritis. Immunol Rev 204: 55-73, 2005

2. Weyand CM, Bryl E and Goronzy JJ. The role of T cells in rheumatoid arthritis. Arch Immunol Ther Exp 48: 429-235, 2000

3. Cope AP. T cells in rheumatoid arthritis. Arthrutis Res Ther 10: S1, 2008

4. Taylor PC. Anti-TNF therapy for rheumatoid arthritis and other inflammatory diseases. Mol Biotechnol 19: 153-168, 2001

5. Olsen NJ and Stein CM. New drugs for rheumatoid arthritis. N Engl J Med 350: 2167-2179, 2004

6. Emery P, Kvien TK, Combe B, Freundlich B, Robertson D, Ferdousi T, Bananis E, Pedersen R and Koenig AS. Combination etanercept and methotrexate provides better disease control in very early ( $<=4$ months) versus early rheumatoid arthritis ( $>4$ months and $<2$ years): post hoc analyses from the COMET study. Ann Rheum Dis 71: 989-992, 2012

7. Brocq O, Millasseau E, Albert C, Grisot C, Flory P, Roux CH and Euller-Ziegler L. Effect of discontinuing TNFalpha antagonist therapy in patients with remission of rheumatoid arthritis. Joint Bone Spine 76: 350-355, 2009

8. Gremese E, Salaffi F, Bosello SL, Ciapetti A, Bobbio-Pallavicini F, Caporal R and Ferraccioli G. Very early rheumatoid arthritis as a predictor of remission: a multicentre real life prospective study. Ann Rheum Dis 72: 858-862, 2013

9. Smolen JS, Aletaha D, Bijlsma JW, Breedveld FC, Boumpas D, Burmester G, Combe B, Cutolo M, de Wit M, Dougados M, Emery P, Gibofsky A, Gomez-Reino JJ, Haraoui B, Kalden J, Keystone EC, Kvien TK, Mclnnes I, Martin-Mola E, Montecucco C, Schoels M, van der Heijde D and T2T Expert Committee. Treating rheumatoid arthritis to target: recommendations of an international task 
force. Ann Rheum Dis 69: 631-637, 2010

10. Mahnke K, Johnson TS, Ring S and Enk AH. Tolerogenic dendritic cells and regulatory $\mathrm{T}$ cells: a two-way relationship. J Dermatol Sci 46: 159-167, 2007

11. Maldonado RA and von Andrian UH. How tolerogenic dendritic cells induce regulatory T cells. Adv Immunol 108: 111-165, 2010

12. Steinman RM, Hawiger D and Nussenzweig MC. Tolerogenic dendritic cells. Annu Rev Immunol 21: 685-711, 2003

13. Alanara T, Karstila K, Moilanen T, Silvennoinen O and Isomaki P. Expression of IL-10 family cytokines in rheumatoid arthritis: elevated levels of IL-19 in the joints. Scand J Rheumatol 39: 118-126, 2010

14. Afzali B, Lombardi G, Lechler RI and Lord GM. The role of T helper 17 (Th17) and regulatory T cells (Treg) in human organ transplantation and autoimmune disease. Clin Exp Immunol 148: 32-46, 2007

15. Oh S, Rankin AL and Caton AJ. CD4+CD25+ regulatory $\mathrm{T}$ cells in autoimmune arthritis. Immunol Rev 233: 97-111, 2010

16. Mottonen M, Heikkinen J, Mustonen L, Isomaki P, Luukkainen R and Lassila O. CD4+ CD25+ T cells with the phenotypic and functional characteristics of regulatory $\mathrm{T}$ cells are enriched in the synovial fluid of patients with rheumatoid arthritis. Clin Exp Immunol 140: 360-367, 2005

17. Cao D, Borjesson O, Larsson P, Rudin A, Gunnarsson I, Klareskog L, Malmstrom V and Trollmo C. FOXP3 identifies regulatory CD25bright CD4+ T cells in rheumatic joints. Scand J Immunol 63: 444-452, 2006

18. Lee JH, Kim TH, Park HE, Lee EG, Jung NC, Song JY, Seo HG, Seung KB, Chang K and Lim DS. Myosin-primed tolerogenic dendritic cells ameliorate experimental autoimmune myocarditis. Car- diovasc Res 101: 203-210, 2014

19. Choo EH, Lee JH, Park EH, Park HE, Jung NC, Kim TH, Koh YS, Kim E, Seung KB, Park C, Hong KS, Kang K, Song JY, Seo HG, Lim DS and Chang K. Infarcted myocardium-primed dendritic cells improve remodeling and cardiac function after myocardial infarction by modulating the regulatory $\mathrm{T}$ cell and macrophage polarization. Circulation 135: 1444-1457, 2017

20. Lim DS, Kang MS, Jeong JA and Bae YS. Semi-mature DC are immunogenic and not tolerogenic when inoculated at a high dose in collagen-induced arthritis mice. Eur J Immunol 39: 1334-1343, 2009

21. Lis $\mathrm{K}$, Kuzawinska $\mathrm{O}$ and Balkowiec-Iskra E. Tumor necrosis factor inhibitors - state of knowledge. Arch Med Sci 10: 1175-1185, 2014

22. Haraoui B, Keystone EC, Thorne JC, Pope JE, Chen I, Asare CG and Leff JA. Clinical outcomes of patients with rheumatoid arthritis after switching from infliximab to etanercept. J Rheumatol 31 : 2356-2359, 2004

23. Lim DS, Kim JH, Lee DS, Yoon CH and Bae YS. DC immunotherapy is highly effective for the inhibition of tumor metastasis or recurrence, although it is not efficient for the eradication of established solid tumors. Cancer Immunol Immunother 56: 1817-1829, 2007

24. Lutz MB. Therapeutic potential of semi-mature dendritic cells for tolerance induction. Front Immunol 3: 123, 2012

25. Stoop JN, Harry RA, von Delwig A, Isaacs JD, Robinson JH and Hikens CM. Therapeutic effect of tolerogenic dendritic cells in established collagen-induced arthritis is associated with a reduction in Th17 responses. Arthritis Rheum 62: 3656-3665, 2010

26. McInnes IB and Schett G. Cytokines in the pathogenesis of rheumatoid arthritis. Nat Rev Immunol 7: 429-442, 2007 
J.Hard Tissue Biology Vol. 28(3): 265-272, 2019 